\title{
Should physicians prescribe cognitive enhancers to healthy individuals?
}

\author{
Cynthia Forlini MA, Serge Gauthier MD, Eric Racine PhD
}

$\mathrm{V}$ arious studies have reported that prescription stimulants (e.g., methylphenidate, dextroamphetamine mixed salts) and other neuropharmaceuticals (e.g., modafinil) are used by some healthy individuals without diagnosed attention deficit disorder to enhance concentration, memory, alertness and mood, a phenomenon often described as " $\operatorname{cog}$ nitive enhancement" or "neuroenhancement.", Recently the term cognitive enhancement has captured the effort to deliberately augment the performance of healthy individuals and has, therefore, been criticized for the implicit assumption that these agents will be beneficial for healthy people. In contrast, the terms "nonmedical use" and "prescription drug abuse" are used in public health literature. ${ }^{3}$ The prevalence of cognitive enhancer use by students on university campuses ranges from $1 \%$ to $11 \%$. $^{3,4}$ This practice raises important ethical and societal questions concerning the freedom of people to engage in cognitive enhancement and whether this is fair practice in academic and professional environments. ${ }^{5}$ Box 1 presents landmarks in the debate surrounding the ethics of cognitive enhancement. ${ }^{1,3-11}$

Physicians are important stakeholders in this debate, given the risks and regulations of prescription drugs and the potential for requests from patients for cognitive enhancers. ${ }^{12,13}$ The underlying question for physicians is not solely whether cognitive enhancement should be legitimized, but what role physicians should play, and under what circumstances they should prescribe these agents to individuals with no medical need, if at all. ${ }^{13}$ Physician obligation to grant, decline or redirect requests for cognitive enhancement has not been discussed extensively. Box 2 contains additional medical context. ${ }^{7,10,14-16}$

Here we argue that uncertain measures of benefits and harms, limited health care resources and professional integrity of physicians are 3 reasons why physicians should not prescribe cognitive enhancers to healthy individuals.

\section{Why "can" is not enough}

Limited normative guidance supports physicians in their response to patient requests for cognitive enhancement. Based on guidance from its Ethics, Law and Humanities Committee, the American Academy of Neurology has stated that prescribing medications for cognitive enhancement is not ethically obligatory or prohibited, and is therefore ethically permissible,$^{10}$ but that refusing to prescribe is also ethically and legally permissible. ${ }^{10}$ Thus, according to the academy, physicians can, but are not obliged to, grant requests for cognitive enhancers.

The American Academy of Neurology was the first professional association to issue specific guidance for physicians on the subject of cognitive enhancement, which has served as a backdrop for further discussion of physician responses and obligations. The academy's position values the physician-patient relationship as the appropriate locus for addressing and responding to such requests. The academy bases its answers to the initial question "Can physicians prescribe medications for cognitive enhancement?" on the moral acceptability of cognitive enhancement within the existing legal and medical frameworks. However, another question is "Should physicians prescribe cognitive enhancers to healthy individuals?" This question asks whether cognitive enhancement is
Competing interests: Serge Gauthier has served as a consultant for Lilly, Sanofi, Pfizer, Janssen and Merck. He has provided expert testimony at a Health Canada meeting on the behalf of Lilly. He has received payment for the development of educational presentations from Merck Canada and is a member of the data safety monitoring board of Bristol-Myers Squibb and Elan. He has also served as scientific advisor for United Bio Source and Servier. None declared for Cynthia Forlini and Eric Racine.

This article has been peer reviewed.

\section{Correspondence to: Eric Racine, eric.racine@ircm.qc.ca}

CMAJ 2013. DOI:10.1503 /cmaj.121508

\section{- Ker POINTS}

- Clinical and social benefits of medications for healthy individuals using certain prescription drugs for cognitive enhancement are not well supported by scientific and professional literature.

- Physicians have a responsibility to distribute finite and shared resources equitably and prudently, which should lead to prioritizing beneficial treatments for patients.

- Diverging perspectives on how cognitive enhancement aligns with medical professional integrity indicate that cognitive enhancement is not yet a general or an accepted medical practice.

- Physicians should seriously consider refusing to prescribe medications for cognitive enhancement to healthy individuals. 
a morally praiseworthy (not solely acceptable) practice for physicians and summons physicians' broader obligations and responsibilities to their profession and the community. ${ }^{17}$

\section{Are there benefits for patients?}

When investigating a patient request for cognitive enhancement, physicians must determine the patient's chief complaint and the benefit he or she expects from a cognitive enhancer. These questions can be answered in a conversation between the physician and patient, as encouraged by the American Academy of Neurology and other ethical frameworks. ${ }^{13}$ However, the physician must determine whether a medication can actually produce the benefit the patient expects. The main concerns here relate to genuine benefits and proper patient information.

The Canadian Medical Association's Code of

Box 1: Landmarks in the ethics debate on cognitive enhancement

- 2000: A prevalence of $16 \%$ for the recreational use of methylphenidate was reported among 283 respondents at an American college. ${ }^{6}$

- 2004: An article was published addressing the ethical issues of cognitive enhancement, ranging from safety to fairness. ${ }^{5}$

- 2007: The British Medical Association published a discussion paper summarizing the ethical issues relevant to cognitive enhancement; no recommendations were made. ${ }^{7}$

- 2008: In a poll published in Nature, $20 \%$ of respondents reported using a medication for cognitive enhancement. ${ }^{1}$ A subsequent commentary discussed how cognitive enhancement might be beneficial for society if investigated and used responsibly. ${ }^{8}$

- 2009: A Canadian commission called for engagement of public and professional stakeholders to better understand and inform medical and nonmedical uses of psychotropic medications. ${ }^{9}$ The Ethics, Law and Humanities Committee of the American Academy of Neurology stated that prescribing medications for cognitive enhancement is ethically permissible. ${ }^{10}$

- 2010/11: The nonmedical use of prescription stimulants for cognitive enhancement among university students was reported to be $1.3 \%$ $11 \%{ }^{3,4}$ An editorial in CMAJ cautioned against rampant use of stimulants for cognitive enhancement and called for university administrations to curtail this practice. ${ }^{11}$

\section{Box 2: Medical context of cognitive enhancement}

Current data indicate that students more commonly obtain stimulants for enhancement purposes from other students (up to $77 \%$ ) than from medical professionals (11\%). ${ }^{14,15}$ Among those who obtain prescriptions, the content of the clinical conversation is unclear (e.g., did the patients feign symptoms of attention-deficit/hyperactivity disorder or clearly request a cognitive enhancer). Cognitive enhancement in the general population is less well characterized, both in terms of prevalence and relation to medical practice. In a physician survey, Hotze and colleagues reported that $61.7 \%$ of respondents $(n=633)$ received requests for cognitive enhancement by healthy individuals monthly or more often, and $36.7 \%$ prescribed medicines for enhancement monthly or more often. ${ }^{16}$ The survey did not specify whether enhancement requests targeted cognitive or other physical attributes. Of the respondents, $12.3 \%$ reported receiving such requests daily. Both the American Academy of Neurology and the British Medical Association have published guidance on how physicians might approach the ethics of cognitive enhancement and requests for medications. ${ }^{7,10}$
Ethics states generally that physicians must "[r]ecommend only those diagnostic and therapeutic services that [they] consider to be beneficial to [their] patient or to others" and that physicians should "[p]rovide [their] patients with the information they need to make informed decisions about their medical care, and answer their questions to the best of [their] ability."." Currently, it is unclear how physicians would fulfill these responsibilities with respect to cognitive enhancers.

The American Academy of Neurology also suggests that the assessment of benefit be based on "relevant medical principles and available evidence, including the pathophysiology of the disease, pharmacologic properties of the medication, studies or case reports in the professional literature or professional experience" in keeping with the evidence base for prescribing off-label treatments for medical conditions. ${ }^{10}$ This permissive position grants cognitive enhancement the status of moral acceptability without clear and decisive evidence. In fact, there is no substantive evidence to support the use of medications such as acetylcholinesterase inhibitors, antidepressants and stimulants as cognitive enhancers in healthy individuals. ${ }^{19-21}$

The use of stimulants is perhaps the best documented example of putative cognitive enhancers. Their effects seem to be most prominent in spatial working memory and long-term declarative memory in lower-performing individuals. ${ }^{21,22}$ However, medical uses of stimulants are associated with risks of dependence, cardiovascular outcomes and psychosis. ${ }^{23}$ These risks may be compounded by outcomes if individuals are unaware of the correct doses and medical contraindications, ${ }^{24}$ risks might also be compounded by factors that have not yet been studied or documented. ${ }^{25}$ Current studies and medical literature have not captured outcomes such as "being more efficient in work or school" or "getting better grades," which are real-world enhancement goals outside of the laboratory environment. How physicians might reconcile such nonstandardized goals with medical indications and whether the ability or inability to meet these types of goals might eventually be turned into a medical condition by enhancement practices remain impending questions. With uncertain benefits and clear harms, it is difficult to support the notion that physicians should prescribe a medication to a healthy individual for enhancement purposes.

\section{What is the effect on health care resources?}

Another question is whether cognitive enhancers should be readily available to all in the same way 
that medically necessary treatments are and, therefore, whether cognitive enhancement is part of the goals of medicine. ${ }^{26}$ The American Academy of Neurology expresses related concerns that "neuroenhancement therapies are likely to be seen as 'lifestyle' drugs and therefore are unlikely to be covered by third-party payers." ${ }^{\prime 10}$ Both physicians and members of the public are reluctant about cognitive enhancers being covered by insurance and public funds. ${ }^{16,27}$ The use of these drugs might therefore be limited to a relatively small segment of the population who can afford to pay for them. ${ }^{10}$ The concern that those who cannot afford the medications for cognitive enhancement will be at a disadvantage is relevant. As well, health care resources are finite, and access could be inequitable even if cognitive enhancement drugs were affordable.

Recent shortages of generic drugs have illustrated that there can be issues in access to affordable medications. In Canada, the Quebec Commission on the Ethics of Science and Technology cautions that an increase in the demand for psycotropic drugs for cognitive enhancement could affect access. ${ }^{9}$

The costs of prescribing cognitive enhancement drugs would also likely require substantial human resources. The American Academy of Neurology recommends full, thorough assessment of patients who request cognitive enhancers. However, the Quebec Commission on the Ethics of Science and Technology is concerned that because a medical consultation is recommended for a patient to potenitally obtain a prescription, and because health and social services are currently hardly able to meet demands, health professionals might be inclined to promote drug therapy by default so as not to leave people without care. ${ }^{9}$

This phenomenon would have a detrimental impact on the handling of legitimate needs of patients already diagnosed by diverting health resources, both pharmacologic and human, toward cognitive enhancement.

Physicians act as gatekeepers to health care resources for their patients and have a stewardship role for all users of the health care system..$^{28}$ Thus, Canadian physicians must promote equitable access to health care resources and use these resources prudently. ${ }^{18}$ Currently, the limited evidence of benefit and professional experience with cognitive enhancement make it difficult to argue that cognitive enhancement is an appropriate use of health care resources. When put into a context of limited health resources, the time spent consulting with patients seeking cognitive enhancers may be to the detriment of patients who need physician care. In summary, the libertarian ethical position articulated by the
American Academy of Neurology may not be a good fit for Canadian health care systems where health care is a public good.

\section{Is cognitive enhancement consistent with medical professional integrity?}

It is unclear at this point if physicians are willing to integrate cognitive enhancement into their clinical practice and if they think it respects their professional integrity. It has been suggested that cognitive enhancement is within the "domain of other socially useful practices that are acceptable to the profession and society." ${ }^{10}$ However, a low response rate to a survey of general practitioners on this topic has led to the hypothesis that "the issues were too foreign to their daily experiences and not perceived as clinically relevant." ${ }^{27}$ In addition, Hotze and colleagues reported that physicians showed "considerable ambivalence around the issue of enhancement" when questioned about their willingness to prescribe a hypothetical enhancement intervention. ${ }^{16}$ In half of the situations, physicians responded that they might prescribe the intervention but with reservations. Likewise, Banjo and colleagues ${ }^{29}$ presented results from a survey of 212 physicians showing that willingness to prescribe existing medications (e.g., methylphenidate, modafinil) for the potential use of enhancement was low (23 on a 7 -point Likert scale). These findings suggest a less favourable involvement of physicians in cognitive enhancement than was believed.

The surveys by Hotze and colleagues ${ }^{16}$ and Banjo and colleagues ${ }^{29}$ also reported that physicians had major concerns about safety, which may explain some of the reticence to prescribe medications for enhancement based on the harms that may befall otherwise healthy individuals. Academic institutions have been invited to lead efforts to prevent stimulant abuse in universities. ${ }^{11}$ However, this approach should not preempt an in-depth conversation about the involvement and integrity of health care professionals.

In contrast to much of the literature, physicians might be inclined to grant patient requests to keep a good relationship with them. ${ }^{30}$ The CMA Code of Ethics provides guidance on the responsibility of physicians to tell their patient when they are presenting "an opinion that is contrary to the generally held opinion of the profession." ${ }^{18}$ These opinions and guidance on how to best uphold professional integrity when dealing with requests for cognitive enhancement indicate that physicians should not pursue a practice as scientifically and ethically contentious as cognitive enhancement. 


\section{Conclusion}

An international bioethics discussion has surfaced on the ethics of cognitive enhancement and the role of physicians in prescribing cognitive enhancers to healthy people. We discussed 3 reasons why positions based on the concept of moral acceptability do not fully capture important considerations related to moral praiseworthiness. Physicians should consider competing obligations, such as the need for evidence of benefit and upholding equitable and prudent use of resources as well as professional integrity, in conjunction with professional guidance. These obligations also need to be considered in light of the changing face of medicine in which physicians can be bypassed by direct-to-consumer availability of medications. We acknowledge that our reflections could be modulated by the evolving state of science and that future advances (e.g., documentation of interindividual differences of positive or negative pharmacologic response to cognitive enhancers) could have an impact on the current discussion..$^{22}$ Given the current state of limited evidence on medical, scientific, social and ethical aspects of cognitive enhancement, we call for greater attention to its appropriateness within existing Canadian health care systems. We hope that our analysis prompts reflection in the Canadian medical community about the international discussions on cognitive enhancement.

\section{References}

1. Maher B. Poll results: look who's doping. Nature 2008:452:674-5.

2. Wilens TE, Adler LA, Adams J, et al. Misuse and diversion of stimulants prescribes for ADHD: a systematic review of the literature. J Am Acad Child Adolesc Psychiatry 2008;47:21-31.

3. Racine E, Forlini C. Cognitive enhancement, lifestyle choice or misuse of prescription drugs? ethics blindspots in current debates. Neuroethics 2010;3:1-14.

4. Franke AG, Bonertz C, Christmann M, et al. Non-medical use of prescription stimulants and illicit use of stimulants for cognitive enhancement in pupils and students in Germany. Pharmacopsychiatry 2011;44:60-6.

5. Farah MJ, Illes J, Cook-Deegan R, et al. Neurocognitive enhancement: What can we do and what should we do? Nat Rev Neurosci 2004:5:421-5.

6. Babcock Q, Byrne T. Student perceptions of methylphenidate abuse at a public liberal arts college. J Am Coll Health 2000;49:143-5.

7. British Medical Association. Boosting your brainpower: ethical aspects of cognitive enhancement [discussion paper]. London (UK): British Medical Association; 2007.

8. Greely H, Sahakian B, Harris J, et al. Towards responsible use of cognitive-enhancing drugs by the healthy. Nature 2008;456:702-5.

9. Commission de l'éthique de la science et de la technologie. Position statement: psychotropic drugs and expanded uses: an ethical perspective. Québec (QC); 2009.

10. Larriviere D, Williams MA, Rizzo M, et al. Responding to requests from adult patients for neuroenhancements: guidance of the ethics, law and humanities committee. Neurology 2009;73: 1406-12.
11. Rosenfield D, Hebert PC, Stanbrook MB, et al. Time to address stimulant abuse on our campuses. CMAJ 2011;183:1345.

12. Chatterjee A. Cosmetic neurology: the controversy over enhancing movement, mentation, and mood. Neurology 2004;63:968-74.

13. Synofzik M. Ethically justified, clinically applicable criteria for physician decision-making in psychopharmacological enhancement. Neureothics 2009;2:89-102.

14. McCabe SE, Teter CJ, Boyd CJ. Medical use, illicit use and diversion of prescription stimulant medication. $J$ Psychoactive Drugs 2006;38:43-56.

15. Barrett SP, Darredeau C, Bordy LE, et al. Characteristics of methylphenidate misuse in a university student sample. Can J Psychiatry 2005;50:457-61.

16. Hotze TD, Shaw K, Anderson EE, et al. "Doctor, would you prescribe a pill to help me ... ?" A national survey of physicians on using medicine for human enhancement. Am J Bioeth 2011;11: 3-13.

17. Racine E. Pragmatic neuroethics: improving treatment and understanding of the mind-brain. Cambridge (MA): MIT Press; 2010.

18. Canadian Medical Association. CMA code of ethics. Ottawa (ON): The Association; reviewed 2012. Available: www.cma.ca /code-of-ethics (accessed 2012 Nov. 20).

19. Repantis D, Laisney O, Heuser I. Acetylcholinesterase inhibitors and memantine for neuroenhancement in healthy individuals: a systematic review. Pharmacol Res 2010;61:473-81.

20. Repantis D, Schlattmann P, Lainsey O, et al. Antidepressants for neuroenhancement in healthy individuals: a systematic review. Poiesis Prax 2009;6:139-74.

21. Repantis D, Schlattmann P, Laisney O, et al. Modafinil and methylphenidate for neuroenhancement in healthy individuals: a systematic review. Pharmacol Res 2010;62:187-206.

22. Smith ME, Farah MJ. Are prescription stimulants "smart pills"? The epidemiology and cognitive neuroscience of prescription stimulant use by normal healthy individuals. Psychol Bull 2011; 137:717-41.

23. Godfrey J. Safety of therapeutic methylphenidate in adults: a systematic review of the evidence. J Psychopharmacol 2009;23: 194-205.

24. Sussman S, Pentz MA, Spruijt-Metz D, et al. Misuse of "study drugs:" prevalence, consequences, and implications for policy. Subst Abuse Treat Prev Policy 2006;1:15.

25. Volkow ND. Long-term safety of stimulant use for ADHD: findings from nonhuman primates. Neuropsychopharmacology 2012;37:2551-2.

26. Juengst E. What does enhancement mean? In: Parens E, editor. Enhancing human traits: ethical and social implications. Washington (DC): Georgetown Univerity Press; 1998. p. 29-47.

27. Bergström LS, Lynoe N. Enhancing concentration, mood and memory in healthy individuals: an empirical study of attitudes among general practitioners and the general population. Scand $J$ Public Health 2008;36:532-7.

28. Cheshire W. Just enhancement. Ethics Med 2012;26:7-10.

29. Banjo OC, Nadler R, Reiner PB. Physician attitudes towards pharmacological cognitive enhancement: safety concerns are paramount. PLOS ONE 2010;5:e14322.

30. Asscher EC, Bolt I, Schermer M. Wish-fulfilling medicine in practice: a qualitative study of physician arguments. J Med Ethics 2012;38:327-31

Affiliations: Integrated Program in Neuroscience (Forlini), McGill University; Neuroethics Research Unit (Forlini, Racine), Institut de recherches cliniques de Montréal; McGill Center for Studies in Aging (Gauthier), McGill University; Departments of Medicine and of Social and Preventive Medicine (Racine), Université de Montréal; Departments of Neurology and Neurosurgery (Racine), Medicine \& Biomedical Ethics Unit, McGill University, Montréal, Que.

Contributors: All of the authors contributed to the conception of this article. Cynthia Forlini drafted the manuscript, which was revised by all authors. All of the authors approved the final version submitted for publication.

Acknowledgments: The authors thank Isabelle Chouinard for comments on an earlier draft of the manuscript and Allison Yan for administrative support. 